\title{
Corpus callosum agenesis-abnormal genitalia syndrome
}

INSERM

\section{Source}

INSERM. (1999). Orphanet: an online rare disease and orphan drug data base. Corpus callosum agenesis-abnormal genitalia syndrome. ORPHA:2508

Corpus callosum agenesis-abnormal genitalia syndrome is a rare, genetic developmental defect during embryogenesis syndrome characterized by agenesis of the corpus callosum, mild to severe neurological manifestations (intellectual disability, developmental delay, epilepsy, dystonia), and urogenital anomalies (hypospadias, cryptorchidism, renal dysplasia, ambiguous genitalia). Additionally, skeletal anomalies (limb contractures, scoliosis), dysmorphic facial features (prominent supraorbital ridges, synophris, large eyes) and optic atrophy have been observed. 